\title{
A MAGNETIC QUADRUPOLE PICK-UP FOR THE CERN PS.
}

\author{
A. Chapman-Hatchett, A. Jansson ${ }^{* \dagger}$, D.J. Williams \\ PS Division, CERN, CH-1211 Geneva 23, Switzerland.
}

\begin{abstract}
In the LHC era, there will be a need to monitor and correct betatron mismatch between machines in a non-destructive way. For this purpose, a quadrupole pick-up has been designed for the CERN PS. Originally, the PS was built for much larger beam sizes than now required when generating the LHC beam, but its large physical aperture should be maintained. Because of this large aperture to beam-size ratio, the quadrupole signal component in a standard pick-up design is strongly suppressed with respect to the commonmode signal, and thus demands a very high common-mode rejection in the signal processing. A magnetic quadrupole pick-up has been designed, in which the common-mode rejection is incorporated in the pick-up itself, by virtue of its geometry. The rejection is thus limited only by mechanical tolerances and can therefore be very large. Without the common-mode component, the dominating signal is dipolar, and small when the beam is centred in the pick-up. The dipole and quadrupole signals can thus be separated using a hybrid circuit with only moderate demands on commonmode rejection. Another advantage with a magnetic pickup is the low output impedance, allowing the use of passive components when forming the quadrupole signal, which is highly desirable in the radiation environment of the PS ring. Simulations and tests of the pick-up and read-out electronics, as well as the first results with real beam are presented.
\end{abstract}

\section{INTRODUCTION}

\subsection{Why a Quadrupole Pick-Up?}

In the production of the LHC beam, the rms emittance blow-up at each inter-machine interface has to be kept lower than $10 \%$. This is less than the absolute accuracy of any present diagnostic tool for emittance measurement [1]. Thus, a comparison of emittance measurements made in two consecutive machines along the injection chain is not enough. If the injection mismatch causing the blow-up can be measured directly, a higher sensitivity can be achieved since many error sources are eliminated.

A quadrupole pick-up measures the aspect ratio $Q=\sigma_{x}^{2}-\sigma_{y}^{2}$ of the beam. At least two individual pickups are needed to estimate the horizontal and vertical beam emittances (if the beta function is known) and six pick-ups are needed to measure the emittance and Twiss parameters of the beam in both planes [2].

\footnotetext{
* CERN and Manne Siegbahn Laboratory, Stockholm, Sweden.
}

† Email: Andreas.Jansson@cern.ch
If the beam size is oscillating in one plane, for example due to optical mismatch at injection, the quadrupole pick-up signal will be modulated by twice the betatron frequency. Since the tune is generally different in the horizontal and vertical planes, the oscillations in the two planes can be separated in a Fourier transform of the signal. Therefore, a single quadrupole pick-up is useful as a diagnostic tool for beam optical matching between machines.

\section{THEORETICAL CONSIDERATIONS}

\subsection{Electric Coupling}

The traditional design of a quadrupole pick-up couples to the electric field of the beam. The electric potential $V(r, \theta)$ around an infinitely long beam is given by

$$
\begin{gathered}
V(r, \theta)=m_{00} \frac{1}{r}+m_{01} \frac{\cos \theta}{r^{2}}+m_{10} \frac{\sin \theta}{r^{2}}+ \\
\quad+m_{11} \frac{\cos 2 \theta}{r^{3}}+\left(m_{20}-m_{02}\right) \frac{\sin 2 \theta}{r^{3}}+\cdots,
\end{gathered}
$$

where the moments $m_{i j}$ of the transverse charge distribution $\rho(x, y)$ are defined as

$$
m_{i j}=\int \rho(x, y) x^{i} y^{j} d x d y
$$

and the order of each moment is $k=i+j$. If electrodes are positioned at $\theta=0, \pi / 2, \pi, 3 \pi / 2$, the quadrupole signal $Q$ can be obtained by

$$
Q=V\left(r_{p}, 0\right)-V\left(r_{p}, \frac{\pi}{2}\right)+V\left(r_{p}, \pi\right)-V\left(r_{p}, \frac{3 \pi}{2}\right),
$$

where $r_{p}$ is the radius of the pick-up. The individual signals are dominated by the first term in (1), which is of the order $m_{0 \mathrm{th}} / r_{p}=\rho_{\text {tot }} / r_{p}$, whereas the quadrupole signal component in each signal is of the order $m_{2 \mathrm{nd}} / r_{p}^{3}=\rho_{t o t} \sigma^{2} / r_{p}^{3}$. Thus, there is factor $\sigma^{2} / r_{p}^{2}$ difference in signal strength. In the case of the LHC beam in the PS ring, where the beam size is much smaller than the aperture, this demands a very high common-mode rejection for the derivation of $Q$, since a difference between two large signals has to be taken.

\subsection{Magnetic Coupling}

The magnetic field around an infinite beam has two components. If the field is expressed in cylindrical coordinates, the $\theta$-component $B_{\theta}$ is essentially given by (1) but with an extra factor $\mu_{0} / 2 \pi$ [3]. The $B_{r}$-component

$$
\begin{array}{r}
B_{r}(r, \theta)=m_{01} \frac{\mu_{0}}{2 \pi} \frac{\sin \theta}{r^{2}}+m_{10} \frac{\mu_{0}}{2 \pi} \frac{\cos \theta}{r^{2}}+ \\
+m_{11} \frac{\mu_{0}}{2 \pi} \frac{\sin 2 \theta}{r^{3}}+\left(m_{20}-m_{02}\right) \frac{\mu_{0}}{2 \pi} \frac{\cos 2 \theta}{r^{3}}+\cdots
\end{array}
$$


is more interesting. In this case, there can be no $m_{00}$ component since this would violate Maxwells equation $\nabla \cdot B=0$. The rest of the formula is similar, however. Therefore, if a pick-up is set up to measure only $B_{r}$, there is no intensity signal. Furthermore, if the beam is centred in the pick-up the dipole signal vanishes, and thus a magnetic pick-up coupling to $B_{r}$ measures "only" the quadrupole signal. A coupling to the radial field component $B_{r}$ is achieved by placing the coupling loops in the plane of constant $r$ (in cylindrical coordinates).

\subsection{Masking of the quadrupole oscillation sig- nal due to dipole oscillation}

A problem with all quadrupole pick-ups is that if the pickup and beam centre do not coincide, the quadrupole signal gets an extra contribution proportional to the square of the displacement. If the displacement is large relative to the beam size, this will dominate the measured quadrupole signal. But, since this pick-up also measures the displacement of the beam, it should be possible to correct for the dipole component, providing the measurement is accurate enough.

\section{PROTOTYPE PICK-UP DESIGN}

\subsection{Mechanical Design}

A prototype pick-up coupling to the radial magnetic field of the beam has been built and installed in the PS machine during the 98/99 winter shutdown. The mechanical design of the prototype pick-up has been based on a spare ceramic vacuum chamber designed for the PS Booster position monitors, whose diameter of $145 \mathrm{~mm}$ fits the physical aperture of the PS machine. The ceramic allows the coupling loops to be placed outside the vacuum, which saves vacuum feed-throughs and facilitates possible interventions once the pick-up is installed in the machine. The loops are placed directly on the outside of the ceramic, at radius $r_{p}=80 \mathrm{~mm}$. The $\theta$-range covered by each loop was chosen to be $45^{\circ}$, which was found to be a good compromise. A wider angle would give larger coupling to the beam but also increase the inter-loop coupling. To avoid build-up of static charges, and to reduce the longitudinal impedance seen by the beam at higher frequencies [4], the inside of the ceramic chamber was coated with a few microns of titanium. For continuity of low frequency wall currents, and to protect against outside noise, the whole arrangement is housed in a metal cylinder of $300 \mathrm{~mm}$ diameter. This shield also enhances the quadrupole signal relative to the dipole signal and reduces the inter-loop couplings.

\subsection{Read-Out Electronics}

The copper rods that make up the loops are connected to earth at one end, and connected in pairs to 1:15 transformers at the other end. The secondary side of each transformer is connected via $50 \Omega$ cable to a hybrid circuit, which is

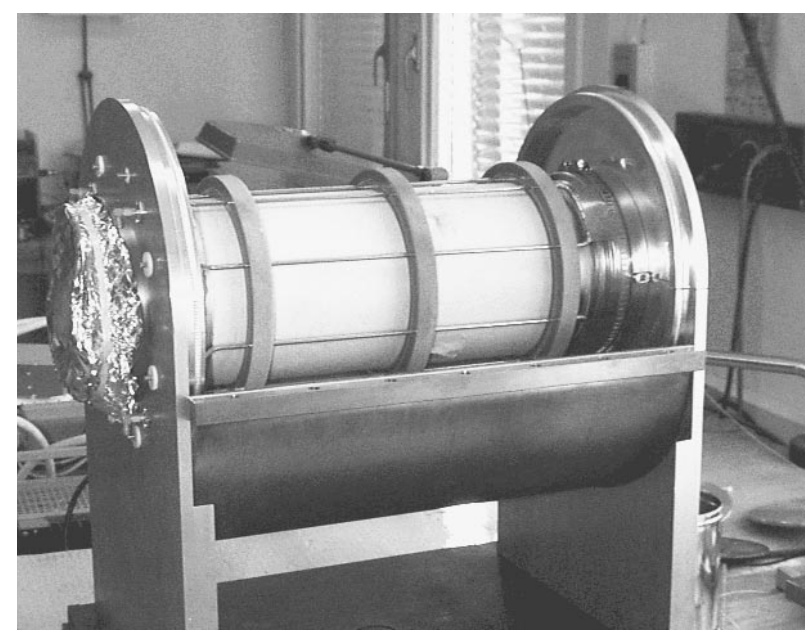

Figure 1: A picture of the pick-up before mounting the shield cylinder.

physically separated from the pick-up to facilitate modifications. The load seen by each antenna loop is thus only $225 \mathrm{~m} \Omega$, which gives a low cut-off frequency of around $100 \mathrm{kHz}$.

The hybrid forms the four composite signals; quadrupole, the two dipoles, and the sum (which ideally should be zero). It can be bypassed using remotely controlled switches, in case the signals from the pick-up are to be measured individually. The hybrid outputs are fed to amplifiers, which are placed under the floor to protect them from radiation. These amplifiers have an an amplification factor of 100, a bandwidth of $30 \mathrm{Mhz}$, and a noise level of $2.3 \mathrm{nV} / \mathrm{Hz}^{-1 / 2}$.

\section{TESTS AND MEASUREMENTS}

\subsection{Simulations}

Simulations of the pick-up have been performed using the High Frequency Structure Simulator (HFSS) program, using a slightly simplified model for the pick-up geometry. The results show, as expected, that the sum signal is totally suppressed. The different couplings obtained from these simulations are shown in Table 1.

\begin{tabular}{|c|cc|}
\hline Moment & Dipole & Quadrupole \\
\hline Analytic (w/o coating) & $10 \mathrm{nH} / \mathrm{mm}$ & $180 \mathrm{pH} / \mathrm{mm}^{2}$ \\
Simulation (w. coating) & $1.5 \mathrm{nH} / \mathrm{mm}$ & $32 \mathrm{pH} / \mathrm{mm}^{2}$ \\
\hline
\end{tabular}

Table 1: Coupling strengths from analytic approximation and simulation.

\subsection{Test Bench Measurements}

Test bench measurements have been performed, using a wire antenna to map the signal as a function of an- 
tenna position in the $x-y$ plane. The result is shown in Figures. 2 and 3. Typical output signals in time domain are shown in Figure. 4. Some ringing at the first resonance frequency of the cavity, formed by the shielding cylinder, was found at about $70 \mathrm{MHz}$. The signal is a common mode signal, and is therefore suppressed by the hybrid. It will also be filtered out by the limited bandwidth of the amplifiers. It was experimentally verified that, thanks to the titanium layer, the beam does not see a significant longitudinal impedance at the resonance.

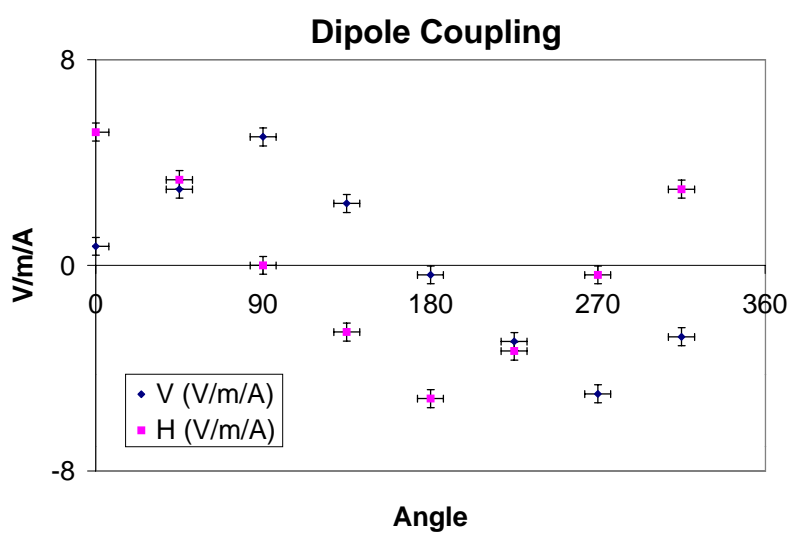

Figure 2: Output dipole signals versus azimuthal angle of antenna in the transverse plane. Antenna displacement from centre: $3 \mathrm{~cm}$. Note the $\sin \theta$ and $\cos \theta$ structure, which is expected for the dipole signal.



Figure 3: Output quadrupole signal versus azimuthal angle of antenna in the transverse plane. Antenna displacement from centre: $3 \mathrm{~cm}$. Note the $\cos 2 \theta$ variation, which signifies the quadrupole signal.

\section{SUMMARY AND CONCLUSIONS}

A quadrupole pick-up coupling to the radial magnetic field induced by the beam has been built and tested. This pick-up design has several advantages with respect to a 'traditional' design, such as:

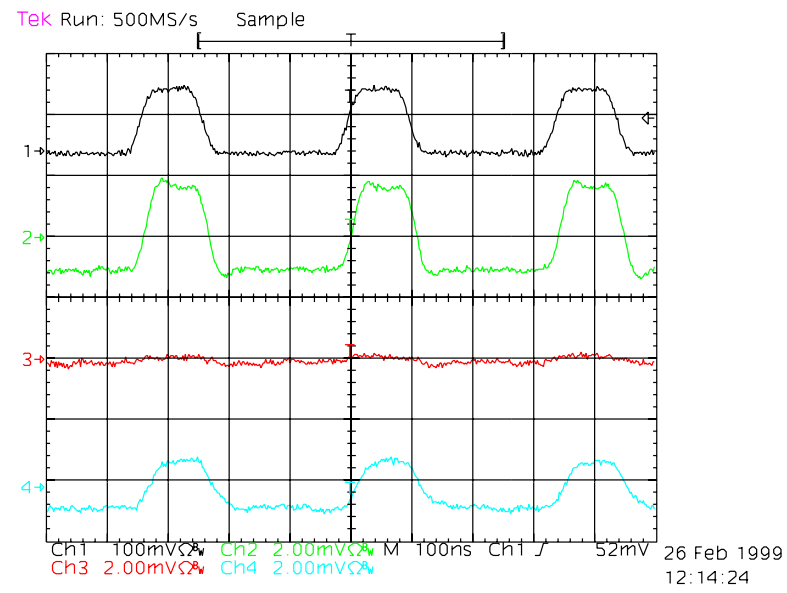

Figure 4: Output signals from the pick-up in time domain. Trace 1 is the input signal, traces 2 and 3 are the horizontal and vertical dipole signals respectively. Trace 4 is the quadrupole signal. The test was performed with a wire antenna displaced $3 \mathrm{~cm}$ horizontally.

- Suppression of the dominating sum signal, thus facilitating the signal treatment.

- No active electronic components needed in the radiation environment of the ring.

- No vacuum feed-throughs necessary.

The test bench measurements have shown that the pick-up works as expected. It will be thoroughly tested with beam during the coming year.

\section{ACKNOWLEDGEMENTS}

The authors would like to thank F. Caspers, J.M. Roux and L. Soby for practical help and interesting discussions.

\section{REFERENCES}

[1] H. Koziol, K Wittenburg, "Summary on Transverse Emittance Measurements and Instruments", Proceedings of the 4th ICFA Beam Dynamics Mini-Workshop on Transverse Emittance Preservation and Measurement, CERN, 1997, CERN/PS/DI/Note98-03, CERN Internal Note.

[2] R.H. Miller, J.E. Clendenin, M.B. James, J.C. Sheppard, "Non-Intercepting Emittance Monitor", Proceedings of the XII High Energy Accelerator Conference, 1983.

[3] A. Jansson, "The Measurement of Higher Order Moments of Beam Transverse Distribution Using a Magnetic Pick-Up.", 1998, CERN/PS/OP/Note98-27, CERN Internal Note.

[4] C. Gonzalez, M. Morvillo, M. D’yachkov, "Impedance Measurements on the LHC Dump Kicker Prototype", LHC Project Note 151. 